
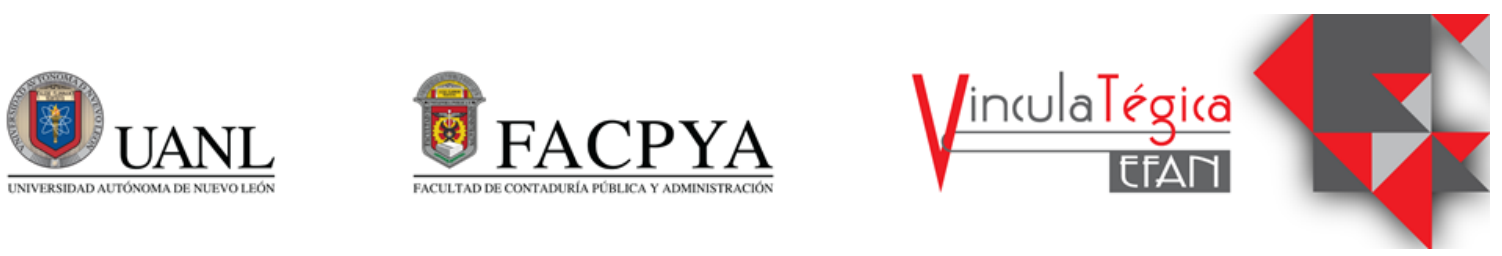

\title{
Censo por estratificación tarifaria y tipo de usuario del sistema intermunicipal de aguas y saneamiento de Monclova-Frontera
}

\author{
Laura Leticia Gaona Tamez ${ }^{1}$, Blanca Estela Montano Pérez² y Gabriel Aguilera Mancilla ${ }^{3}$ \\ ${ }^{1}$ Universidad Autónoma de Coahuila, Facultad de Contaduría y Administración Monclova, Coahuila, México \\ lauragaonatamez@uadec.edu.mx, Carretera 57 Km 4.5 Monclova, Coahuila, (+52) 8661331949 \\ ${ }^{2}$ Universidad Autónoma de Coahuila, Facultad de Contaduría y Administración Monclova, Coahuila, México \\ blancamontanoperez@uadec.edu.mx $x^{2}$ Carretera 57 Km 4.5 Monclova, Coahuila, (+52) 8661354290 \\ ${ }^{3}$ Universidad Autónoma de Coahuila, Facultad de Contaduría y Administración Monclova, Coahuila, México, \\ gabo76mancilla@hotmail.com ${ }^{3,}$ Carretera 57 Km 4.5 Monclova, Coahuila, (+52) 8661554353
}

Información del artículo revisado por pares

Fecha de aceptación: junio-2021

Fecha de publicación en línea: diciembre-2021

DOI: https://doi.org/10.29105/vtga7.1-151

\section{Resumen}

El objetivo de este proyecto es realizar un censo para obtener, además de otros datos, información estadística, pertinente y actualizada sobre la ubicación y condición de cada uno de los predios por contrato y de su correspondiente status tarifario en Monclova y Frontera, Coahuila. Esto, con la finalidad de atender la solicitud planteada a la Facultad de Contaduría y Administración (FCA) por el Sistema Intermunicipal de Aguas y Saneamiento de Monclova-Frontera (SIMAS), un organismo público descentralizado intermunicipal. Así mismo, este censo permitirá al Cuerpo Académico obtener información sobre las empresas familiares existentes en estas dos ciudades conurbadas. La presente, será una investigación de campo con un enfoque cuantitativo, emplea el método estadísticodescriptivo y el diseño no experimental - transversal. Se elaborará la cédula o ficha censal y se administrará a un total de 82, 455 usuarios que conforman el padrón de SIMAS las cuales hasta el momento de la investigación no se han aplicado. Posteriormente, los datos recabados serán procesados estadísticamente a través del Paquete Estadístico Aplicado a las Ciencias Sociales (SPSS). Los resultados obtenidos permitirán incrementar la eficiencia administrativa de SIMAS y para el C. A. resultará de considerable valor, para sus investigaciones empíricas, contar con una base de datos sobre la cantidad y características de las empresas familiares existentes en éstas dos ciudades
The objective of this project is to carry out a census to obtain, in addition to other data, statistical, pertinent and updated information on the location and condition of each of the properties under contract and their corresponding rate status in Monclova and Frontera, Coahuila. This, in order to meet the request made to the Faculty of Accounting and Administration (FCA) by the Inter-municipal Water and Sanitation System of Monclova-Frontera (SIMAS), an inter-municipal decentralized public body. Likewise, this census will allow the Academic authority to obtain information on the existing family businesses in these two metropolitan cities. This will be a field investigation with a quantitative approach, uses the statistical-descriptive method and the non transversal experimental design. The census card or file will be prepared and administered to a total of 82,455 users that make up the SIMAS registry, the time of the investigation have not been applied. Subsequently, the data collected will be statistically processed through the Statistical Package for the Social Sciences (SPSS). The results obtained will make it possible to increase the administrative efficiency of SIMAS and for C. A. it will be of considerable value, for its empirical research, to have a database on the number and characteristics of existing family businesses in these two metropolitan cities. 
conurbadas.

Palabras clave: Censo, estratificación tarifaria, SIMAS

\section{INTRODUCCIÓN}

La ausencia de un plan de desarrollo urbano equilibrado y sustentable, aunado al vertiginoso incremento de nuevos asentamientos poblacionales en Monclova y Frontera constituye para SIMAS, un reto. Pues existe la necesidad de contar con estadísticas e indicadores confiables, pertinentes y actualizados sobre el status tarifario, condición de cada uno de sus usuarios y de los predios que se tienen registrados.

Al momento de la elaboración del presente documento, las ciudades de Monclova y Frontera se encuentran conformadas por 115,431 predios, de los cuales SIMAS tiene un padrón de 89,177 cuentas. En ese sentido, existen 87,905 predios dibujados y ligados a una cuenta, lo que representa un avance del $98.6 \%$.

SIMAS estratificó y agrupó en 20 sectores comerciales a la población de usuarios de Monclova y Frontera.

Así mismo, SIMAS cuenta con un Padrón que muestra la clasificación de la cantidad de usuarios de acuerdo con cada tarifa/giro.

Con la finalidad de atender y dar respuesta a la necesidad planteada por SIMAS a la FCA de contar, a través de un censo, con información actualizada de la población total de usuarios sobre su condición predial y asignación tarifaria.

Por lo antes expuesto el objetivo de proyecto es realizar un censo para obtener, mediante una herramienta confiable
Keywords: Census, tariff stratification, SIMAS

JEL: J10, H00, H49, H40.

información estadística, pertinente y actualizada sobre la ubicación y condición de cada uno de los predios por contrato y de su correspondiente status tarifario. Así mismo, a través del mismo proyecto el cuerpo Académico podrá integrar, como resultado de este censo, una base de datos confiable sobre la cantidad y características de las empresas familiares existentes en Monclova y Frontera, Coahuila, su giro, tamaño y ubicación.

\section{MARCO TEÓRICO}

En estadística descriptiva se define al censo, como un padrón de personas que integran una localidad, también se define como el grupo de datos de referencia a observar. Un censo recopila datos sobre las características de una población Como tal, el censo no trabaja sobre una muestra, sino sobre la población total.

Desde la perspectiva de la investigación científica, se considera al censo como una técnica que emplea como instrumento para la recopilación de datos la cédula o ficha censal (Wallgren, A. y Wallgreen, B., 2011).

\subsection{Historia de los censos}

Desde que el hombre empezó a integrarse en sociedades cada vez más complejas, vio la necesidad de contabilizar los miembros que constituían su comunidad, con el objetivo de conseguir el abastecer de alimentos para todos. Esto lo hacían por medio de marcas en piedras simbolizando a las personas, animales cazados y productos recolectados, usando diferentes símbolos para en cada caso. 
Al pasar del tiempo, aparecieron las grandes civilizaciones de la historia y con esto el recuento de los pobladores con objetivos militares, ya que se necesitaba salvaguardar las fronteras e incluso extenderlas.

En la Biblia, el libro "Números", que forma parte del Pentateuco, hace mención a un censo de personas y grupos o familias ("tribus"); en él se halla una lista de todos los individuos que salieron de Egipto con Moisés.

En el Nuevo Testamento, al narrar la historia del nacimiento de Jesús existe una reseña explícita de la realización de censos que era habitual en el imperio romano: cada cinco años se llevaba a cabo un "census" de habitantes, patrimonios y de individuos en condiciones de integrarse al ejército.

Cuando el Imperio romano cayó se dejaron de hacer los censos. Se realizaron algunos conteos parciales de los sirvientes y de las tierras. Para conocer el daño provocado por la peste, dieron inicio a un registro de los fallecimientos causados por la enfermedad (De León, L., 2012).

\subsection{Historia de los censos en México}

En 1895 en el gobierno de Porfirio Díaz, se vivió en México una época de estabilidad política, social y económica. Es cuando se efectúa el primer censo del México independiente por la Dirección General de Estadística encabezada por Antonio Peñafiel. Aunque no se contaba con evidencias documentales sobre censos anteriores del diseño conceptual, es posible que la contenido considerado se haya basado en censos de otros países, principalmente de Francia y los Estados Unidos, ajustándolos a las necesidades propias de México. La fecha del levantamiento censal fue el 20 de octubre de 1895 .

El procesamiento de la información se realizó de manera manual, lo que represento una enorme carga de trabajo, con muchas limitaciones para poder aprovechar la información recabada, por esta razón no se mostrarían datos para todas las variables contenidas en las cédulas censales y ningún cruzamiento de variables.

Para dar a conocer los resultados, los cuales tardaron 4 años en estar listos, con los que se realizó una publicación del total del país y varios documentos por entidad federativa, lo cual involucró un esfuerzo descomunal para las condiciones técnicas existentes. Así, en 1899 México situó la publicación de resultados del primer censo de población (INEGI, 2009).

\subsection{Surgimiento de los censos económicos}

$\mathrm{Al}$ inicio del siglo $\mathrm{XX}$ se ejecutaron algunos esfuerzos apartados para la producir información estadísticas referente a la situación economía nacional, destacando los resultados que obtuvo la entonces Secretaría de Industria, Comercio y Trabajo en la segunda década.

Debido a la falta de información estadística que señalara el grado de desarrollo económico en el país y la necesidad de mostrar la distribución agraria que repercutió de la Revolución Mexicana dando origen al decreto presidencial del 6 de junio de 1929, expedido por el Lic. Emilio Portes Gil, Presidente de la República, en el cual se expuso el beneficio social de los censos de población, agrícola, ganadero e industrial. El decreto instituía, entre otras cosas, que el padrón industrial se haría el 15 de octubre de 1929 y el censo, el 15 de mayo de 1930.

En 1930 se inició con los Censos Económicos, comenzando únicamente con el manufacturero. Con el continuo desarrollo económico y urbano del país se requería contar con estadísticas más recientes, y como resultado, se instituyó la repetición quinquenal de los Censos Económicos (INEGI, 2009).

\subsection{Antecedentes}


SIMAS Monclova - Frontera es el sistema de Agua y Saneamiento que suministra a las poblaciones de estos municipios proveyendo los servicios de agua, alcantarillado y saneamiento, para uso comercial, doméstico e industrial.

SIMAS es un organismo público descentralizado intermunicipal para los municipios de Monclova y Frontera Coahuila, con personalidad jurídica y patrimonio propios. Fue creado por el Decreto 300 y publicado en el Diario Oficial (DOF) el 31 de Agosto de 1993, por el entonces gobernador del Estado De Coahuila el C. Lic. Eliseo Francisco Mendoza Berrueto (DOF, 1993).

El objeto de este organismo denominado SIMAS es; "administrar, operar, conservar, mantener, construir y rehabilitar el sistema de agua potable, agua desalada, drenaje, alcantarillado y tratamiento de aguas residuales, correspondiente a los municipios de Monclova y Frontera, Coahuila, así como fijar y cobrar tarifas que se generen por la prestación de dichos servicios" (DOF, 1993). El Sistema de agua potable se obtiene primeramente de corrientes subterráneas agrupadas en el ejido Pozuelos del municipio de Frontera, la cual posee 27 pozos que generan 1,590 litros de agua por segundo, ubicados en el sur y suroeste de la ciudad.

La dirección actual incremento la productividad arrancando un programa de renovación de equipo para asegurar la continuidad del servicio, el programa contempla la renovación de bombas y motores de alta eficiencia reemplazando el equipo de bombeo en los 27 pozos con los que se cuenta para mejorar el servicio, a la vez se modernizaron los centros de control de motores, cambiando los arrancadores que tenían más de 20 o 30 años de uso y que eran de tensión reducida por los de estado sólido que tienen un arranque más suave y con una velocidad progresiva para evitar daños en los equipos.

El agua se transporta desde una distancia de $25 \mathrm{~km}$ por acueductos o líneas de conducción de 24 o 30 pulgadas de diámetro. En su camino, el agua pasa por el sistema de cloración para proceder a su desinfección y posteriormente se almacena en los 17 tanques de almacenamiento distribuidos en las partes altas de la ciudad.

La misión de SIMAS es:
"proporcionar servicios de agua,
alcantarillado y saneamiento para uso
doméstico, comercial e industrial, con
calidad, costo razonable, en forma eficiente y
efectiva".

Su visión es "ser un sistema de agua y saneamiento de excelencia, reconocido por su alta competitividad e innovación constante, la confiabilidad de su personal y la confianza de la ciudadanía".

Con fundamento en su misión y visión SIMAS planteó a la Facultad de Contaduría de la Universidad Autónoma de Coahuila la necesidad de actualizar, a través de un censo, los datos del padrón de usuarios con el fin establecer una equidad tarifaria entre los mismos.

\section{5 objetivos del proyecto}

El proyecto que en este documento se presenta tiene como finalidad: Atender y dar respuesta a la necesidad planteada por SIMAS a la FCA de contar, a través de un censo, con información actualizada de la población total de usuarios sobre su condición predial y asignación tarifaria.

Así mismo, a través del mismo proyecto se obtendrá como resultado de este censo, una base de datos confiable sobre la cantidad y características de las empresas familiares existentes en Monclova y Frontera, Coahuila, su giro, tamaño y ubicación. El cual será de gran utilidad para cuerpo académico.

En la Tabla 1 se presentan los objetivos generales de SIMAS y del cuerpo académico. En la Tabla 2 se muestran los 
objetivos específicos de SIMAS y del cuerpo

académico.

Tabal 1: Objetivos Generales

\begin{tabular}{|c|c|}
\hline SIMAS & $\begin{array}{c}\text { C.A. ADMINISTRACIÓN, DESARROLLO Y } \\
\text { COMPETITIVIDAD DE LAS EMPRESA FAMILIARES }\end{array}$ \\
\hline $\begin{array}{l}\text { Realizar un censo para obtener, mediante una } \\
\text { herramienta confiable información estadística, } \\
\text { pertinente y actualizada sobre la ubicación y } \\
\text { condición de cada uno de los predios por contrato y } \\
\text { de su correspondiente status tarifario. Esto, con el fin } \\
\text { de incrementar la eficiencia operativa de SIMAS a } \\
\text { través de la actualización y renovación del Plano } \\
\text { Digital de SIMAS de acuerdo con los cambios } \\
\text { urbanísticos de Monclova-Frontera. }\end{array}$ & $\begin{array}{l}\text { Aprovechar la infraestructura disponible y los recursos } \\
\text { humanos para determinar cuántas empresas familiares existen } \\
\text { en Monclova y Frontera, Coahuila }\end{array}$ \\
\hline
\end{tabular}

Fuente: elaboración propia.

Tabla 2: Objetivos Específicos

\begin{tabular}{|c|c|}
\hline SIMAS & $\begin{array}{c}\text { C.A. ADMINISTRACIÓN, DESARROLLO Y } \\
\text { COMPETITIVIDAD DE LAS EMPRESA FAMILIARES }\end{array}$ \\
\hline $\begin{array}{l}\text { - Actualizar y/o corregir los datos } \\
\text { domiciliarios de cada contrato y de su } \\
\text { asignación tarifaria. } \\
\text { Identificar y clasificar la ubicación de los } \\
\text { giros y nombres mercantiles de } \\
\text { instituciones, empresas, asociaciones, etc. } \\
\text { - Categorizar la condición de cada uno de los } \\
\text { predios por contrato: habitado, deshabitado, } \\
\text { en ruinas, terreno baldío o área verde. } \\
\text { Registrar la condición de la toma por } \\
\text { diámetro y tipo de material. } \\
\text { Obtener datos actualizados y confiables } \\
\text { sobre el medidor por cada uno de los } \\
\text { contratos. } \\
\text { Detectar el tipo de almacenamiento: } \\
\text { cisterna, tinaco, ambas o por abasto directo. } \\
\text { Ubicar la localización del drenaje y registro. } \\
\text { Identificar y señalar el material del que está } \\
\text { hecha la banqueta. } \\
\text { Actualizar y/o corregir la asignación } \\
\text { tarifaria de acuerdo con el tipo de predio y } \\
\text { contrato: popular, interés social, urbano } \\
\text { medio, residencial, mixto, comercial, } \\
\text { industrial, pública y de beneficencia. } \\
\text { Identificar y detectar la existencia de toma } \\
\text { clandestina. }\end{array}$ & $\begin{array}{l}\text { Obtener un registro de la cantidad de empresas } \\
\text { familiares que existen en Monclova y Frontera, } \\
\text { Coahuila } \\
\text { - Conocer y clasificar el giro de dichas empresas. } \\
\text { - Identificar y clasificar estos negocios de acuerdo con } \\
\text { los rangos de micro, pequeña, mediana y grande } \\
\text { empresa. } \\
\text { Obtener el nombre y ubicación de las empresas } \\
\text { familiares en éstas dos ciudades. }\end{array}$ \\
\hline
\end{tabular}

Fuente: elaboración propia. 


\section{MÉTODO}

Para esta investigación se recurre al enfoque cuantitativo, al método estadísticodescriptivo y al diseño no experimental transversal.

Por enfoque cuantitativo se entiende aquella investigación en la que se recopilan datos numéricos de los objetos, fenómenos o participantes que estudia y analiza mediante procedimientos estadísticos (Hernández-Sampieri, R., FernándezCollado, C. y Lucio, P., 2006).

A través del método estadístico descriptivo se define y reseña un fenómeno señalando sus características, interrelaciones de los hechos y los cambios que supone en el transcurso del tiempo. Implica la expresión cuantitativa, por medio de cuadros y gráficas, de las relaciones que guardan los hechos que conforman un fenómeno (Elizondo, A., 2002). cuantitativa, no se manipulan deliberadamente las variables como en la experimental. Es decir, no se hace variar intencionalmente la (s) variable (s) independiente para medir su efecto en otras variables. Se pretende observar los fenómenos tal como se dan en su contexto natural para un análisis posterior (Sampieri, R., et. al., 2006).

En una investigación bajo el diseño transversal se recopila los datos en un momento único en el tiempo, a diferencia del longitudinal que recaba los datos en diferentes puntos del tiempo (Sampieri, R., et. al., 2006).

SIMAS cuenta con un Padrón que muestra la clasificación de la cantidad de usuarios de acuerdo con cada tarifa/giro. Dicho Padrón se muestra en la Tabla 3.

En la investigación no experimental

Tabla 3: Padrón por tarifa/giro y usuario

\begin{tabular}{|c|c|c|c|c|c|c|}
\hline \multicolumn{3}{|l|}{ TARIFA (GIRO) } & \multicolumn{4}{|c|}{ USUARIO } \\
\hline \multicolumn{3}{|l|}{ Popular } & \multicolumn{4}{|c|}{44,682} \\
\hline \multicolumn{3}{|l|}{ Interés Social } & \multicolumn{4}{|c|}{28,196} \\
\hline \multicolumn{3}{|l|}{ Residencial } & \multicolumn{4}{|c|}{6,233} \\
\hline \multicolumn{3}{|l|}{ Comercial } & \multicolumn{4}{|c|}{1,850} \\
\hline \multicolumn{3}{|l|}{ Industrial } & \multicolumn{4}{|c|}{919} \\
\hline \multicolumn{3}{|l|}{ Público } & \multicolumn{4}{|c|}{383} \\
\hline \multicolumn{3}{|l|}{ Beneficencia } & \multicolumn{4}{|c|}{2} \\
\hline \multicolumn{7}{|l|}{ Fuente: SIMAS (2013) } \\
\hline \multicolumn{7}{|c|}{$\begin{array}{l}\text { Dado que es un censo, no se } \\
\text { procede la extracción de una muestra. Por } \\
\text { tanto, se administrarán } 82,455 \text { fichas o } \\
\text { cédulas censales pertenecientes al padrón de } \\
\text { usuarios de SIMAS, los cuales están } \\
\text { sectorizados en la Tabla } 4 .\end{array}$} \\
\hline \multicolumn{7}{|c|}{ Tabla 4: Padrón de usuarios de SIMAS por sector } \\
\hline Usuarios & Sector & Usuarios & Sector & Usuarios & Sector & Usuarios \\
\hline 3,014 & 6 & 4,816 & 11 & 795 & 16 & 2,407 \\
\hline 5,638 & 7 & 3,633 & 12 & 4,078 & 17 & 4,354 \\
\hline 4,305 & 8 & 3,087 & 13 & 3,960 & 18 & 5,052 \\
\hline
\end{tabular}




\begin{tabular}{cccccccc}
\hline 4 & 4,316 & 9 & 5,678 & 14 & 4,055 & 19 & 3,618 \\
5 & 4,401 & 10 & 4,666 & 15 & 4,887 & 20 & 5,695 \\
& & & & & & & Total 82,455 \\
\hline
\end{tabular}

Fuente: SIMAS (2013)

La Figura 1 muestra la distribución geográfica de los 20 sectores que integran el padrón de SIMAS.

Figura 1: Distribución geográfica de los sectores del padrón de SIMAS

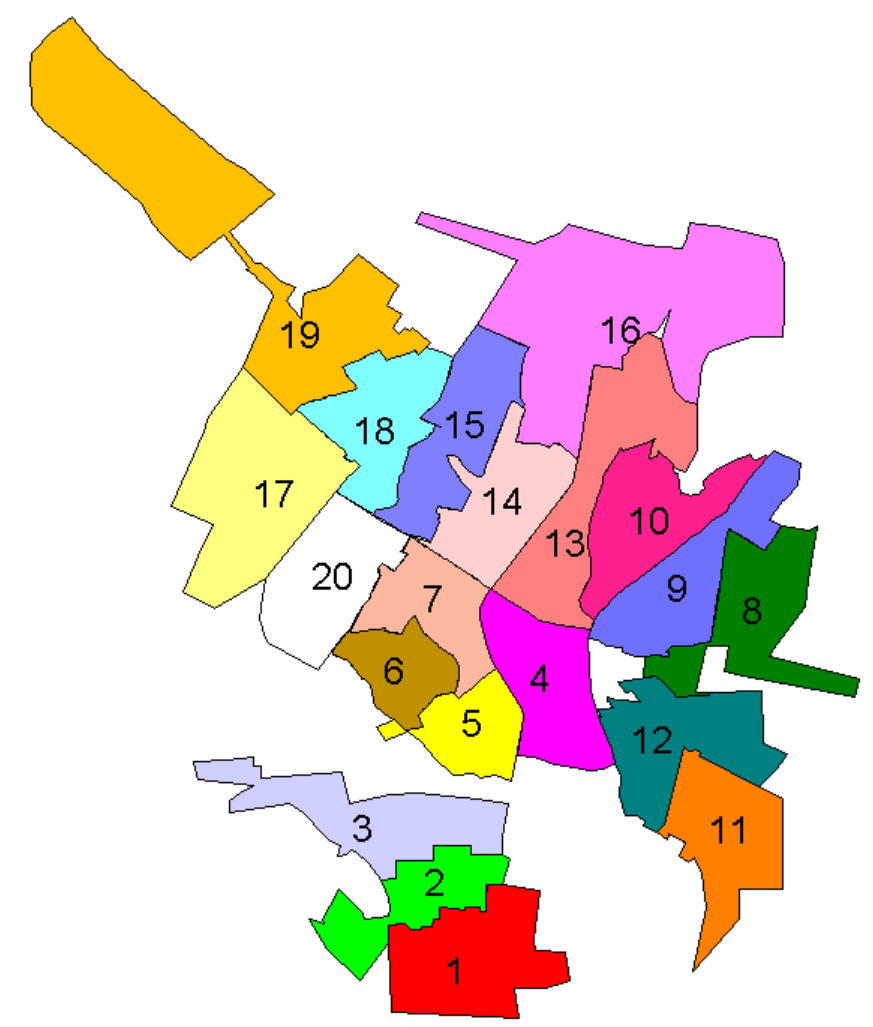

Fuente: SIMAS (2013)

En la Tabla 5 se presentan las variables a medir en la temática censal.

Tabla 5: Variables a medir y/o temática censal

\begin{tabular}{|ccc|}
\hline Tipo de tarifa & Datos del medidor & Giro \\
Dirección correcta & Tipo de almacenamiento & Nombre comercial \\
Condición del predio & Drenaje y material de banqueta & Empresa familiar \\
Condición de la toma & Tipo de predio (Tarifa) & Tamaño de la empresa \\
\hline Fuente: elaboración propia. & & \\
\hline
\end{tabular}


La realización del censo se efectuará

en cuatro etapas que se describen a continuación en la Tabla 6. Los recursos y la infraestructura disponible para la realización de este proyecto se presenta en la Tabla 7.

Tabla 6: Etapas del proceso censal

I Etapa: Planeación Revisión bibliográfica sobre procedimientos para la elaboración de un censo.

Diseño del instrumento (cédula censal)

Prueba piloto

Capacitación de encuestadores

Logística y coordinación de tareas

II Etapa: Recopilación de Trabajo de campo por sectores datos

III Etapa: Codificación, Clasificación de los datos mediante la codificación y tabulación de los tabulación, procesamiento y mismos y su procesamiento para efectuar el análisis correspondiente. análisis de la información.

IV Etapa: elaboración del Redacción del informe final informe de investigación.

Fuente: elaboración propia.

Tabla 7: Descripción de los recursos humanos e infraestructura disponible.

\begin{tabular}{|c|c|c|c|}
\hline $\begin{array}{l}\text { Recurso } \\
\text { Humano }\end{array}$ & Requisitos & Responsabilidades & Cantidad \\
\hline $\begin{array}{l}\text { Catedrático- } \\
\text { investigador }\end{array}$ & $\begin{array}{l}\text { Competencia investigadora: } \\
\text { capacidad para solucionar } \\
\text { problemas en el proceso de } \\
\text { investigación sobre la base de la } \\
\text { versatilidad y flexibilidad } \\
\text { metodológica, conociendo las } \\
\text { posibilidades y limitaciones de } \\
\text { cada estrategia metodológica. }\end{array}$ & $\begin{array}{l}\text { Responsable de cada una de las } \\
\text { etapas del proceso que conlleva la } \\
\text { realización del censo: } \\
\text { Planeación } \\
\text { Recopilación } \\
\text { Procesamiento de datos } \\
\text { Elaboración del Informe Final }\end{array}$ & $\begin{array}{l}04 \\
\text { Catedráticos- } \\
\text { investigadores. } \\
01 \\
\text { Colaborador } \\
\text { del C.A. }\end{array}$ \\
\hline $\begin{array}{l}\text { Población } \\
\text { estudiantil FCA }\end{array}$ & $\begin{array}{l}\text { Estudiantes que decidan } \\
\text { participar responsablemente en } \\
\text { el levantamiento del censo. } \\
\text { Pueden participar de los tres } \\
\text { programas: LAE, CP, y LARH } \\
\text { de quinto semestre en adelante. }\end{array}$ & $\begin{array}{l}\text { Participación en la recolección de } \\
\text { datos bajo la doble supervisión: } \\
\text { SIMAS y catedráticos. } \\
\text { Custodia de la cédula censal. } \\
\text { Apoyo en la captura de datos en el } \\
\text { programa SPSS. } \\
\text { Auxiliar en la elaboración de }\end{array}$ & $\begin{array}{l}26 \text { alumnos de } \\
\text { los diferentes } \\
\text { programas } \\
\text { educativos. }\end{array}$ \\
\hline
\end{tabular}


materiales de trabajo requeridos.

\begin{tabular}{|c|c|c|}
\hline \multirow{3}{*}{$\begin{array}{l}\text { Elementos } \\
\text { materiales }\end{array}$} & \multirow[b]{2}{*}{ Área y equipo para la captura y } & \\
\hline & & 2 escritorios \\
\hline & procesamiento de la & 5 sillas \\
\hline & información & 1 Lap top \\
\hline & & 1 impresora \\
\hline
\end{tabular}

Económicos

Fuente: elaboración propia.

drenaje y registros, materiales utilizados en banquetas.

\section{RESULTADOS}

A través de la realización del Censo por Estratificación Tarifaria y Tipo de Usuario del Sistema Intermunicipal de Aguas y Saneamiento de Monclova-Frontera (SIMAS), se pretende obtener los siguientes beneficios:

- Proveer a la administración de SIMAS datos actualizados para incrementar el insumo de información requerida para un adecuado proceso de toma de decisiones.

- Disminuir y evitar rezagos existentes en cuanto a información sobre cada uno de los 20 sectores.

- Promover la equidad tarifaria de acuerdo con el giro, tipo de construcción, etc.

- Evitar retrasos por parte de las cuadrillas de trabajo por errores actuales en los datos domiciliarios.

- Incrementar la eficiencia administrativa al contar con información actualizada de la ubicación de los giros y nombres mercantiles de empresas, instituciones, etc.

- Obtener estadísticas descriptivas sobre tomas, medidores, tipo de almacenamiento, localización del
- A través de contar con una estadística sobre la existencia de tomas clandestinas, proceder a la disminución de las mismas.

- Mayor conocimiento del usuario.

- Contar con estadísticas que proporcionen un panorama de la dinámica urbana, condición y evolución de los asentamientos poblacionales más recientes.

- Dado que existe una ausencia de información pertinente y actualizada sobre el número y características de las empresas familiares en Monclova y Frontera, resulta de suma importancia y beneficio para el C. A. contar con esta base de datos para realizar mayores y mejores investigaciones empíricas.

\section{CONCLUSIONES}

A diferencia otras investigaciones donde se emplea la muestra, el censo consiste principalmente en, una investigación de campo.

Como tal, esto implica formular y calendarizar un plan de acción que establezca las actividades a realizar y el tiempo en que estas serán ejecutadas. De tal forma que cada una de las etapas que conlleva la realización de un censo se convierte en un objetivo particular de acción (Babbie, E., 2000; Wallgren, A., y Wallgren, B., 2011; INEGI, 1999). 
Cabe mencionar que el proyecto aún no ha sido aprobado es solamente la propuesta presentada a Simas, el cual una vez aprobado, se tendrían como metas:

1. A los tres primeros meses se habrá concluido con la etapa de planeación: revisión bibliográfica, diseño de la cédula censal, realización de la prueba piloto, capacitación de los encuestadores y definición de la logística y tareas a realizar.

2. A partir del cuarto mes se procederá a la realización del trabajo de campo para el llenado de 82,455 cédulas censales, esto tomará 18 meses de trabajo.

3. De manera simultánea se procederá a la captura de datos y procesamiento de la información.

4. En los últimos tres meses se elaborará él informa final y se darán a conocer los resultados a SIMAS.
La Facultad de Contaduría y Administración (FCA) de la Universidad Autónoma de Coahuila (UA de C) como promotor de actividades de investigación con impacto en la sociedad asumió, a través del C.A. Administración, Desarrollo y Competitividad de las Empresas Familiares, presentar el proyecto de realizar para el Sistema Intermunicipal de Aguas y Saneamiento de Monclova-Frontera (SIMAS), el censo por estratificación tarifaria y tipo de usuario de SIMAS.

Esto, con la finalidad de mejorar la eficacia $y$ eficiencia de los procesos administrativos, operativos, de servicio y cobranza de SIMAS; así como para actualizar y mejorar el Plano Digital de SIMAS respecto a los cambios urbanísticos en Monclova y Frontera, Coahuila.

La intención de esta investigación es presentar la propuesta del proyecto que por el momento va en la etapa de revisión de bibliografía. 


\section{REFERENCIAS}

Ander Egg, E., (2003), Técnicas de investigación social., Editorial El Ateneo S.A. de C.V. México.

Babbie, E. (2000), Fundamentos de la investigación social., International Thomson Editores. México.

De León, L. (2012). La Estadística. Recuperado de: http://www.monografias.com/trabajos91/acerca-estadistica/acerca-estadistica.shtml

Diario Oficial 300 (1993) Decreto 300, Martes 31 de Agosto.

Elizondo, A., (2002), Metodología de la investigación contable. Thomson, México.

Instituto Nacional de Estadística y Geografía INEGI (2011), Micro, pequeña y gran empresa: estratificación de los establecimientos: censos económicos 2009. Recuperado de: http://www.inegi.org.mx/est/contenidos/espanol/proyectos/censos/ce2009/pdf/Mono_Micro _peque_mediana.pdf

INEGI, (2011), Metodología de los censos económicos 2009. Recuperado de: http://www.inegi.org.mx/est/contenidos/espanol/metodologias/censos/CE09_Metodologia.p df

Instituto Nacional de Estadística y Geografía INEGI, I Censo de captación, tratamiento y suministro de agua. Censos económicos 1999. Recuperado de: http://www.inegi.org.mx/prod_serv/contenidos/espanol/bvinegi/productos/censos/economic os/1999/suministro de agua/rdagua.pdf

Levin, J. y Levin, C., (2011), Fundamentos de estadística en la investigación social. Alfaomega y Oxford, México.

Ponce-Rodas (2005), Técnicas de procesamiento de datos en censos y encuestas. Universidad de San Carlos. Recuperado de: http://biblioteca.usac.edu.gt/tesis/08/08_0240_CS.pdf

Sampieri R., Fernández-Collado, C., Lucio, P. (2006), Metodología de la investigación. Cuarta Edición. Mc Graw Hill, México.

Vallejo, A., Procesamiento informático de los censos. XI Censo de Población y Vivienda 1990. XI Censo de Población y Vivienda 1990. Recuperado de: http://www.juridicas.unam.mx/publica/librev/rev/rap/cont/80/pr/pr6.pdf

Wallgren, A., y B., Wallgren., (2011), Encuestas por muestreo, censo y encuestas por registros. Oficina de estadística y Universidad de Örbro. Curso sobre el aprovechamiento estadístico de los registros administrativos. INEGI. Recuperado de:

http://ceacepalrtc.org/download files/Descripci\%C3\%B3n\%20curso\%20sobre\%20Registro s\%20administrativos.pdf 